\section{Cuarta Reunión Anual de la Sociedad Latinoamericana de Fisiología Respiratoria 2020. Premiación de Trabajos libres}

\author{
Fourth Annual Meeting of \\ the Latin American Society of \\ Respiratory Physiology 2020, \\ SOLAFIRE. Abstracts awards
}

\author{
Ireri Isadora Thirión-Romero* \\ *Presidente de la Sociedad Latinoamericana \\ de Fisiología Respiratoria.
}

Citar como: Thirión-Romero II. Cuarta Reunión Anual de la Sociedad Latinoamericana de Fisiología Respiratoria 2020. Premiación de Trabajos libres. Neumol Cir Torax. 2021; 80 (4): 310-312. https:// dx.doi.org/10.35366/103458

El pasado 13 y 14 de noviembre se llevó a cabo la Cuarta Reunión Anual de la Sociedad Latinoamericana de Fisiología Respiratoria 2020. En esa ocasión, por la situación de pandemia, la reunión se realizó de forma virtual. En los cuatro años de vida que lleva la Sociedad Latinoamericana de Fisiología Respiratoria (SOLAFIRE), el número de personas interesadas en la fisiología respiratoria se ha incrementado de la misma manera que se han fortalecido los lazos de colaboración entre los países de Latinoamérica. No hay duda de que la unión hace la fuerza y es precisamente la SOLAFIRE, con el talento de cada uno de sus miembros, quien nos convoca cada año a intercambiar ideas, conceptos, proyectos y un largo etcétera. Continuemos tejiendo sueños para concretarlos en esta vasta región que representamos desde el Río Bravo hasta la Tierra del Fuego.

En esta ocasión compartimos con ustedes los trabajos libres que fueron premiados en la última reunión anual de la SOLAFIRE 2020. Todos los trabajos fueron de mucho interés y de valor científico incuestionable. La selección de los ganadores se llevó a cabo mediante evaluación anónima, analizándose diferentes componentes del trabajo de investigación mediante una lista de cotejo. Felicidades a los ganadores y nuestro agradecimiento a Neumología y Cirugía de Tórax (NCT) por permitirnos compartir con ustedes el trabajo de nuestros asociados.

Correspondencia:

Dra. Ireri Isadora Thirión-Romero

Correo electrónico:

draisadora_thirion@hotmail.com

\section{PRIMER LUGAR}

Evaluación de Pimax y Pemax en pacientes recuperados de COVID-19 en el Instituto Nacional de Enfermedades Respiratorias

$$
\begin{gathered}
\text { Amaury González-Molina, } \\
\text { Laura Gochicoa-Rangel,* } \\
\text { Carlos Guzmán-Valderrábano, } \\
\text { Wilmer, Madrid-Mejía* }
\end{gathered}
$$

Isabel Salas-Escamilla, Mónica Silva-Cerón,* José Christian Rodríguez-Hernández,* Adela Durán-Cuéllar,*

Irlanda de Jesús Alvarado-Amador,* Antonio Salles-Rojas*

*Departamento de Fisiología Respiratoria Instituto Nacional de Enfermedades Respiratorias Ismael Cosío Villegas.

Antecedentes: Hasta 20\% de los pacientes con COVID-19 desarrolla síndrome de dificultad respiratoria aguda, $6 \%$ de ellos requieren manejo en unidad de cuidados críticos e intubación orotraqueal, lo cual ha sido asociado a debilidad muscular. Previamente se ha reportado la alteración en Pimax y Pemax en pacientes recuperados de COVID-19. Objetivos: Informar los resultados de la presión inspiratoria y espiratoria máxima (Pimax y Pemax) al mes y a los tres meses posteriores al egreso del INER tras haber presentado COVID-19. Material y métodos: Estudio descriptivo longitudinal; se incluyeron pacientes del Instituto Nacional de Enfermedades Respiratorias recuperados de COVID-19. Se les realizaron pruebas de Pimax y Pemax y se dividieron en dos grupos: intubados y no intubados. Se utilizó la prueba de Mann-Whitney para comparación entre ambos grupos y prueba t pareada para comparar ambas visitas. Resultados: Se incluyeron 33 pacientes, la media de edad fue 44.87 años, 11 ameritaron intubación orotraqueal; $27 \%$ presentaba Pimax menor de $80 \%$ del predicho en la primera visita comparado con $21 \%$ en la segunda visita. En Pemax, 81\% presentaba valores debajo de $80 \%$ del predicho en la primera visita y $72 \%$ en la segunda visita. No se encontraron diferencias significativas en Pimax $(p=0.88 ; p=0.56$, inicial y seguimiento respectivamente) y Pemax $(p=0.88$; $p=0.56$ ) entre pacientes intubados $y$ no intubados. La prueba de t pareada para visita 1 y visita 2 mostró cambios estadísticamente significativos para Pimax $(p=0.02)$, no así para Pemax $(p=0.75)$. Conclusiones: Este trabajo destaca la importancia de la debilidad de la musculatura respiratoria en pacientes recuperados de COVID-19, predominantemente manifestado por la prueba de Pemax, independientemente del antecedente de intubación orotraqueal. Ambas pruebas tienen un valor clínico significativo en el seguimiento de este grupo de pacientes.

\section{SEGUNDO LUGAR}

$$
\begin{gathered}
\text { Precisión de los espirómetros } \\
\text { ultrasónicos } \mathrm{NDD}^{\circledR} \text { al agregar } \\
\text { diferentes filtros de alta eficiencia } \\
\text { Irlanda de Jesús Alvarado-Amador,* } \\
\text { Laura Graciela Gochicoa-Rangel, } \\
\text { CarlosGuzmán-Valderrábano }
\end{gathered}
$$

*Neumología Pediátrica. Instituto Nacional de Enfermedades Respiratorias Ismael

Cosío Villegas; ${ }^{\ddagger}$ Departamento de Fisiología Respiratoria. Instituto Nacional de Enfermedades Respiratorias Ismael Cosío Villegas.

Los espirómetros Easy One ${ }^{\circledR}$ NDD $^{\circledR}$ utilizan un sensor de flujo/volumen ultrasónico que permite utilizar consumibles desechables sin necesidad de un filtro viral/bacteriano. Debido a la posible generación de aerosoles de las PFR, actualmente las diferentes sociedades internacionales recomiendan la realización de éstas utilizando filtros bacterianos/virales con eficacia mínima probada para flujos 
espiratorios de 600-700 L/min, por ello la empresa NDD ${ }^{\circledR}$ ha recomendado el uso de un adaptador que permite agregar un filtro de alta eficiencia $\left(V_{B M a x}{ }^{\circledR} 35 \mathrm{~mm}\right.$ A-M Systems), pero se desconoce si la precisión en las mediciones puede verse afectada con el uso de éste u otros filtros virales-bacterianos. Objetivo: Comparar la precisión de las mediciones espirométricas agregando cinco diferentes filtros de alta eficiencia. Material y métodos: Estudio transversal, comparativo y observacional. Filtros utilizados: Eco-BVF ${ }^{\circledR}$, MicroGard- II ${ }^{\circledR}, V_{B M a x}{ }^{\circledR}$, Sibelmed ${ }^{\circledR}$ y Filtrette $^{\circledR}$. Se eligieron aleatoriamente 10 de las 50 curvas de volumen y flujo de la American Thoracic Society (ATS) y se reprodujeron utilizando diferentes combinaciones colocando los filtros en el extremo proximal y distal del Spirette ${ }^{\circledR}$ conectado a un espirómetro EasyOne PC, se utilizó como control el Spirette ${ }^{\circledR}$ sin otro filtro realizando mismas curvas. Los límites de precisión para FEV1, FVC y PEF fueron los recomendados por los estándares de la ATS y se consideró una precisión recomendable una tasa de fallo menor de 5\%. Resultados: 300 observaciones con diferentes combinaciones de filtros y las curvas seleccionadas. En las 30 mediciones con el Spirette ${ }^{\circledR}$ sin filtro, todas las curvas se encontraron dentro de los rangos de precisión aceptables por el estándar a diferencia del resto de las combinaciones, en las cuales en todas se encontró una tasa de error superior a 5\% (mediana 58.33\% - Mín: 36.67\%, Máx: 75.56\%). Conclusión: Agregar filtros de alta eficiencia a los espirómetros ultrasónicos EasyOn-PC modifica de manera importante la precisión de las mediciones de la espirometría.

\section{TERCER LUGAR}

Cambios en la función pulmonar de los pacientes recuperados de COVID-19 medido por espirometría, difusión de monóxido de carbono y caminata de 6 minutos

\section{W Madrid-Mejía, \\ C Guzmán-Valderrábano,* A Salles,* \\ A González,* M Silva,* C Rodríguez,* \\ L Lozano,* M López,* L Gochicoa-Rangel*}

*Instituto Nacional de Enfermedades Respiratorias Ismael Cosío Villegas.
Antecedentes: Se sabe poco de las secuelas funcionales de la COVID-19, la mayoría de los estudios de función pulmonar posCOVID-19 son descriptivos y sólo existe un único prospectivo realizado por Kai Liu et al. en China, donde se les realizó capacidad de difusión pulmonar de monóxido de carbono (DLCO), espirometría y prueba de caminata de seis minutos (PC6M) basal y a las seis semanas, observando mejoría en las pruebas de función pulmonar (PFR) luego de la rehabilitación. Objetivo: Describir el cambio de la función pulmonar por espirometría, DLCO y PC6M en pacientes recuperados de COVID-19 al mes y a los tres meses de haber sido dados de alta. Material y métodos: Se hizo una cohorte de pacientes dados de alta del INER, donde se les realizó espirometría, DLCO y PC6M al mes y a los tres meses. Resultados: Hasta el momento son 38 sujetos con el seguimiento hasta el tercer mes, se compararon las pruebas de función pulmonar (PFR) al mes y a los tres meses encontrando diferencia $(p<0.000)$ en la DLCO (aumento) sin observar diferencias en la PC6M y espirometría, pero sí se detectaron anormalidades en las pruebas realizadas. En las espirometrías se observó: normal en $75 \%$, sugestivo de restricción $17.5 \%$ y $3 \%$ con obstrucción. En la caminata presentaban menos metros caminados de su predicho y alrededor de la mitad de los pacientes mostraban un criterio de desaturación durante la prueba. Conclusiones: Existen modificaciones en las pruebas de función pulmonar observando una mejoría de $2.4 \mathrm{~mL} / \mathrm{mmHg} / \mathrm{min}$ de la DLCO a tres meses del alta.

\section{MENCIÓN ESPECIAL}

Diferencias en función pulmonar acorde con la gravedad de COVID-19

$$
\begin{gathered}
\text { Stephanie López-Romero, } \\
\text { Diana Lizbeth Ortiz-Farías, } \\
\text { Roberto Mancilla-Ceballos, } \\
\text { Esperanza Figueroa-Hurtado, } \\
\text { Saúl Vázquez-López, } \\
\text { Yuri Noemí Pou-Aguilar, } \\
\text { Arturo Cortés-Telles }{ }^{\ddagger}
\end{gathered}
$$

*Departamento de Medicina Interna; *Departamento de Neumología y Cirugía de Tórax. Hospital Regional de Alta Especialidad de la Península de Yucatán. Mérida, Yucatán. México.
Introducción: Con base en información de otros coronavirus se ha reportado que persisten alteraciones en la función pulmonar por meses e incluso años. Sin embargo, la información relacionada en COVID-19 es escasa y nula en población latina/mexicana. El objetivo es describir las anormalidades de la función pulmonar acorde con la gravedad del padecimiento por COVID-19. Material y métodos: Estudio transversal que incluye a pacientes recuperados de COVID-19. Se clasificaron en cuatro grupos acorde con la gravedad del padecimiento. A cada paciente se le aplicó un cuestionario de síntomas y las siguientes pruebas: espirometría simple, difusión pulmonar de monóxido de carbono (DLCO) y caminata de seis minutos (C6M) con base en los procedimientos estándares vigentes. Las variables continuas se presentan como promedios con desviación estándar (DE), en tanto las categóricas como frecuencias con porcentajes. La comparación entre grupos se realizó mediante el análisis de varianza de una sola vía para mediciones múltiples seguido de un ajuste post hoc con Bonferroni para variables continuas. Se consideró como estadísticamente significativo un valor de $p$ menor de $5 \%$ bimarginal. Resultados: Se evaluaron 158 pacientes con edad promedio de $48 \pm 14$ años. A mayor gravedad de la enfermedad mayor proporción del sexo masculino. No se observaron diferencias entre los grupos en la persistencia de síntomas. Con base en espirometría y DLCO observamos una tendencia lineal e inversamente proporcional, a mayor gravedad menor FVC $(p<0.001)$ y DLCO $(p=0.011)$. Respecto a la C6M, los casos leves recorrieron la mayor distancia (505 \pm 77 $\mathrm{m})$, en tanto los casos críticos tuvieron una mayor proporción de pacientes con una tasa de recuperación cardíaca subóptima en el minuto 1 (heart rate [HHR1] $<12$ latidos, $p=0.047$ ). Conclusiones: Las anormalidades en la función pulmonar en el período posCOVID-19 tienen una relación lineal e inversamente proporcional acorde con la gravedad al inicio de la enfermedad.

\section{MENCIÓN ESPECIAL}

Síntomas y función pulmonar a las 6 semanas de egreso por COVID-19

Stephanie López-Romero, ${ }^{*}$ Roberto Mancilla-Ceballos, ${ }^{*}$ Esperanza Figueroa-Hurtado, 
Neumol Cir Torax. 2021; 80 (4): 310-312

\section{Diana Lizbeth Ortiz-Farías, Saúl Vázquez-López, Yuri Noemí Pou-Aguilar, Arturo Cortés-Telles ${ }^{\ddagger}$}

*Departamento de Medicina Interna; ${ }^{\ddagger}$ Departamento de Neumología y Cirugía de Tórax. Hospital Regional de Alta Especialidad de la Península de Yucatán. Mérida, Yucatán. México.

Introducción: En COVID-19 escasos reportes han descrito la persistencia de síntomas y anormalidades en la función pulmonar; sin embargo, la información relacionada en población latina/mexicana es nula. El objetivo es describir y contrastar la frecuencia de síntomas y función pulmonar a las seis semanas de egreso en pacientes recuperados de COVID-19. Ma- terial y métodos: Estudio transversal que incluye a pacientes que fueron evaluados al egreso por COVID-19. En cada visita se contabilizó la diferencia en semanas desde el egreso hasta la evaluación ( $<$ seis semanas versus $>$ seis semanas) que incluyó un cuestionario de síntomas más espirometría simple y difusión pulmonar de monóxido de carbono con base en las recomendaciones técnicas vigentes. Las variables continuas se presentan como medias con $\mathrm{DE}$, en tanto las categóricas como frecuencias con porcentajes. En el contraste de grupos se realizó un análisis de t-Student de muestras independientes (variables continuas) y la prueba de $\chi^{2}$ (variables categóricas). Se consideró como estadísticamente significativo un valor de p menor de 5\% bimarginal. Resultados: Se evaluaron 73 pacientes con un pro- medio de edad de $48 \pm 13$ años y $40 \%$ fueron mujeres. De las morbilidades, $54 \%$ tenían obesidad y $18 \%$ obesidad grado III. En general, el promedio del número de síntomas persistentes fue $3 \pm 2$ y $31 \%$ manifestó cinco o más síntomas, entre ellos, fatiga ( $73 \%)$, disnea (36\%), tos (34\%), mialgias/artralgias (34\%) fueron los más comunes. Al contrastar los grupos no se observaron diferencias en edad, sexo y función pulmonar mediante espirometría; sin embargo, los casos con menos de seis semanas de egreso mostraron una disminución significativa de la DLCO (68.6\%p versus $78.9 \% p, p=0.043)$. Conclusiones: En población latina/mexicana luego de seis semanas de egreso, tres de cada 10 casos persisten con cinco o más síntomas en el período posCOVID-19 y tienen mayor compromiso en la DLco. 